\title{
MHD Flow with Hall Current and Ion-Slip Effects due to a Stretching Porous Disk
}

\author{
Faiza M. N. El-Fayez \\ Department of Mathematics, Princess Nora Bint Abdul Rahman University, P.O. Box 104502, Riyadh 11636, Saudi Arabia \\ Correspondence should be addressed to Faiza M. N. El-Fayez; dr.faiza5@hotmail.com
}

Received 26 June 2013; Accepted 30 September 2013

Academic Editor: Md Sazzad Chowdhury

Copyright ( 2013 Faiza M. N. El-Fayez. This is an open access article distributed under the Creative Commons Attribution License, which permits unrestricted use, distribution, and reproduction in any medium, provided the original work is properly cited.

\begin{abstract}
A partially ionized fluid is driven by a stretching disk, in the presence of a magnetic field that is strong enough to produce significant hall current and ion-slip effects. The limiting behavior of the flow is studied, as the magnetic field strength grows indefinitely. The flow variables are properly scaled, and uniformly valid asymptotic expansions of the velocity components are obtained. The leading order approximations show sinusoidal behavior that is decaying exponentially, as we move away from the disk surface. The twoterm expansions of the radial and azimuthal surface shear stress components, as well as the far field inflow speed, compare well with the corresponding finite difference solutions, even at moderate magnetic fields. The effect of mass transfer (suction or injection) through the disk is also considered.
\end{abstract}

\section{Introduction}

The flow due to a stretching surface is an important field of fluid mechanics. It has several applications, for example, in production of glass and paper sheets, drawing of plastic films, and extrusion of metals and polymers. When the surface is stretching radially from a point, in a linear manner, the flow is axisymmetric. The velocity components tend to their limits monotonically, in an exponential manner, as we move away from the surface [1]. Recently, the problem and variants thereof have received considerable attention by Ariel [2-5], Ariel et al. [6], and Hayat and coworkers [7, 8]. Different methods of solution and analysis were applied to cases with or without slip conditions, MHD flow, or second-grade fluid. All these cases exhibited the same monotonic behavior.

One important variant is when the fluid is electrically conducting and a magnetic field is applied normally to the surface. For weak magnetic fields, the flow remains axisymmetric. The Lorentz force acts to restrain the flow, causing faster exponential tendency to the limits. When the magnetic field is strong enough to produce significant Hall current, the problem changes considerably. The Hall current is associated with an electromagnetic force which drives an azimuthal flow. The problem loses its axisymmetric nature, maintaining its rotational symmetry, though, with the flow variables being independent of the azimuthal angle.

It is of interest to explore the nature of this MHD flow taking into consideration the Hall current. To that end, the limiting behavior of the flow as the magnetic field grows indefinitely is studied. The straightforward perturbation analysis leads to secular behavior, which is removed by parameter straining [9]. Three-term uniformly valid asymptotic expansions are, thus, obtained. The presence of the Hall current leads to an exponential tendency to the limits but of sinusoidal nature. This behavior is not altered by including mass transfer through the surface or the electromagnetic effect of ion slip. The flow involves alternating regions of forward and backward velocity components.

Finite difference solutions are also obtained and show qualitative adherence to the predicted limiting behavior even for moderate magnetic fields. Quantitatively, the two-term expansions show excellent agreement with the numerical results.

\section{Formulation of the Problem}

A partially ionized fluid is driven by an insulated disk, which is axisymmetrically stretching with speed $u_{s}$ that 
is proportional to the radial distance $r$ from its axis of symmetry $z$. Specifically, $u_{s}=\omega r$, where $\omega$ is a constant of proportionality. The disk is porous, allowing a uniform fluid injection of speed $w_{s}$ in the $z$ direction. Otherwise, the fluid would have been quiescent. A uniform magnetic field is applied in the $z$-direction. The magnetic Reynolds number is small, so that the induced magnetic field can be neglected and the applied field maintains its uniform magnetic flux density $B$. On the other hand, the magnetic field is strong enough to produce significant curvature in the electrons trajectories, leading to considerable Hall currents. Moreover, the electron motion is dominated by electron-ion collisions, so that the ion-slip effect cannot be overlooked [10].

The fluid is incompressible of density $\rho$, viscosity $\mu$, electrical conductivity $\sigma$, Hall coefficient $h\left(=1 / e n_{e}\right)$, and ion-slip coefficient $k\left(=1 /\left(1+n_{e} / n_{a}\right) \kappa_{a i}\right)$, all of which are considered constant. Respectively, $n_{e}$ and $n_{a}$ are the number densities for the electrons and neutral particles, $\kappa_{a i}$ is the coefficient of friction between ions and neutral particles, and $-e$ is electron charge.

The flow is governed by the continuity and Navier-Stokes equations [10]

$$
\begin{gathered}
\nabla \cdot \mathbf{V}=0, \\
\rho \mathbf{V} \cdot \nabla \mathbf{V}=\mu \nabla^{2} \mathbf{V}-\nabla p+\mathbf{J} \times \mathbf{B},
\end{gathered}
$$

where $p$ is the pressure, $\mathbf{V}$ is the velocity vector, $\mathbf{B}$ is the magnetic field, and $\mathbf{J}$ is the electric current. According to the generalized Ohm's law, $\mathbf{J}$ is given by

$$
\mathbf{J}=\sigma[\mathbf{V} \times \mathbf{B}-h \mathbf{J} \times \mathbf{B}-k \mathbf{B} \times(\mathbf{J} \times \mathbf{B})],
$$

where terms on the right-hand side are due to the effects of Lorentz force, the Hall current, and the ion slip, respectively.

Making use of the rotational symmetry, we formulate the problem for a typical meridional plane. The velocity components: $u$ in the $r$-direction, $v$ in the azimuthal direction, and $w$ in the $z$-direction, as well as the pressure $p$, are dependent on $r$ and $z$ only. The governing equations become

$$
\begin{gathered}
u_{, r}+\frac{u}{r}+w_{, z}=0 \\
\rho\left(u u_{, r}-\frac{v^{2}}{r}+w u_{, z}\right)=\mu\left[u_{, r r}+\left(\frac{u}{r}\right)_{, r}+u_{, z z}\right] \\
-p_{, r}-\frac{\sigma B^{2}}{n^{2}+m^{2}}(n u-m v), \\
\rho\left(u v_{, r}+\frac{u v}{r}+w v_{, z}\right)=\mu\left[v_{, r r}+\left(\frac{v}{r}\right)_{, r}+v_{, z z}\right] \\
\quad-\frac{\sigma B^{2}}{n^{2}+m^{2}}(n v+m u), \\
\rho\left(u w_{, r}+w w_{, z}\right)=\mu\left[w_{, r r}+\frac{1}{r} w_{, r}+w_{, z z}\right]-p_{, z},
\end{gathered}
$$

where subscripts following a comma denote differentiation. The Hall parameter $m=\sigma h B$ may be positive or negative in accordance with the sign of $B$, that is, depending on whether the magnetic field is directed away from or toward the disk. However, as a simultaneous change of the signs of $m$ and $v$ leaves the problem unaltered, only nonnegative values of $m$ need to be considered. The parameter $n=1+\sigma k B^{2}$ reduces to unity for zero ion slip.

At the surface, $z=0$, the adherence conditions $u=\omega r$ and $v=0$ apply, together with the injection condition $w=w_{s}$. Far from the disk, as $z \sim \infty$, the fluid has pressure $p_{\infty}$ and velocity components $u \sim 0$ and $v \sim 0$.

The problem admits the similarity transformations $z=$ $(\mu / \rho \omega)^{1 / 2} \zeta, u=\omega r F(\zeta), v=\omega r G(\zeta), w=(\omega \mu / \rho)^{1 / 2} H(\zeta)$, and $p=p_{\infty}+\omega \mu Q(\zeta)$, leading to the following problem:

$$
\begin{gathered}
2 F+H^{\prime}=0, \\
F^{\prime \prime}-H F^{\prime}-F^{2}+G^{2}-\beta(\widehat{n} F-\widehat{m} G)=0, \\
G^{\prime \prime}-H G^{\prime}-2 F G-\beta(\widehat{n} G+\widehat{m} F)=0, \\
Q^{\prime}=H^{\prime \prime}-H H^{\prime}, \\
F(0)=1, \\
G(0)=0 \\
H(0)=H_{s}, \\
F(\infty) \sim 0 \\
G(\infty) \sim 0 \\
Q(\infty) \sim 0
\end{gathered}
$$

where a dash denotes differentiation with respect to $\zeta, \beta=$ $\sigma B^{2} / \rho \omega$ is the magnetic interaction number, and $(\widehat{m}, \widehat{n})=$ $(m, n) /\left(m^{2}+n^{2}\right)$.

\section{Asymptotic Analysis}

We are interested in the limiting behavior of the flow as $\beta \sim \infty$ with fixed $\widehat{m}$ and $\widehat{n}$. That $F(0)=1$ irrespective of the value of $\beta$ means that $F=\mathcal{O}\left(\beta^{0}\right)$. The leading term in (3b) is $\beta \widehat{n} F$. It can be balanced by the diffusion term $F^{\prime \prime}$ in a contracting region in which $\zeta=\mathcal{O}\left(\beta^{-1 / 2}\right)$. Then, $(3 \mathrm{a})$ gives $H=\mathcal{O}\left(\beta^{-1 / 2}\right)$, consistent with which $H_{s}$ must be $\mathcal{O}\left(\beta^{-1 / 2}\right)$. Equation (3d), then, gives $Q=\mathcal{O}\left(\beta^{0}\right)$. In (3c), the driving force for the azimuthal flow is the Hall effect expressed by $\beta \widehat{m} F$. This requires $G^{\prime \prime} \sim \beta \widehat{m} F$, leading to $G=\mathcal{O}\left(\beta^{0}\right)$.

New scaled variables $\eta=\beta^{1 / 2} \zeta$ and $V=\beta^{1 / 2} H$ are introduced, transforming Problem (3a), (3b), (3c), (3d), (3e), $(3 \mathrm{f}),(3 \mathrm{~g}),(3 \mathrm{~h}),(3 \mathrm{i})$, and $(3 \mathrm{j})$ to the form

$$
\begin{gathered}
2 F+V^{\prime}=0, \\
F^{\prime \prime}-\widehat{n} F+\widehat{m} G=\beta^{-1}\left(V F^{\prime}+F^{2}-G^{2}\right), \\
G^{\prime \prime}-\widehat{n} G-\widehat{m} F=\beta^{-1}\left(V G^{\prime}+2 F G\right), \\
Q^{\prime}=V^{\prime \prime}-\beta^{-1} V V^{\prime}, \\
F(0)=1, \\
G(0)=0,
\end{gathered}
$$




$$
\begin{aligned}
& V(0)=V_{s}, \\
& F(\infty) \sim 0, \\
& G(\infty) \sim 0, \\
& Q(\infty) \sim 0,
\end{aligned}
$$

where, now, the dashes denote differentiation with respect to $\eta$.

We expand the flow variables in powers of $\beta^{-1}$ in the form

$$
Z \sim Z_{0}+\beta^{-1} Z_{1}+\beta^{-2} Z_{2}+\cdots
$$

where $Z$ stands for $F, G, V$, and $Q$. The problems for $Z_{n}, n=$ $0,1,2,3, \ldots$, are linear. For $Z_{0}$, we get the solutions [11]

$$
\begin{gathered}
F_{0}=e^{-\alpha \eta} \cos \gamma \eta, \\
G_{0}=-e^{-\alpha \eta} \sin \gamma \eta, \\
V_{0}=V_{s}-\frac{2\left[\alpha+e^{-\alpha \eta}(\gamma \sin \gamma \eta-\alpha \cos \gamma \eta)\right]}{\alpha^{2}+\gamma^{2}}, \\
Q_{0}=-2 e^{-\alpha \eta} \cos \gamma \eta,
\end{gathered}
$$

where $\alpha$ and $\gamma$ satisfy $\alpha^{2}-\gamma^{2}=\widehat{n}$ and $2 \alpha \gamma=\widehat{m}$. For $Z_{1}$, the solutions involve secular terms of the form $\eta e^{-\alpha \eta} \sin \gamma \eta$ and $\eta e^{-\alpha \eta} \cos \gamma \eta$, the removal of which is effected by straining the parameters $\widehat{m}$ and $\widehat{n}$ in the form (5), and the procedure can be continued to higher orders [9].

The following expansions up to $\mathcal{O}\left(\beta^{-2}\right)$ are obtained:

$$
\begin{gathered}
\widehat{n} \sim\left(\alpha^{2}-\gamma^{2}\right)+\beta^{-1} \alpha v_{0}+\beta^{-2} \alpha v_{1}+\cdots, \\
\widehat{m} \sim 2 \alpha \gamma+\beta^{-1} \gamma v_{0}+\beta^{-2} \gamma v_{1}+\cdots, \\
F \sim E C+\beta^{-1}\left[E\left(C f_{1}+S g_{1}\right)-E^{2} f_{1}\right] \\
+\beta^{-2}\left[E\left(C f_{2}+S g_{2}\right)+E^{2} \bar{c}+E^{3}(C \bar{a}+S \bar{b})\right]+\cdots, \\
G \sim-E S+\beta^{-1}\left[E\left(C g_{1}-S f_{1}\right)-E^{2} g_{1}\right] \\
+\beta^{-2}\left[E\left(C g_{2}-S f_{2}\right)+E^{2} \widetilde{c}+E^{3}(C \widetilde{a}+S \widetilde{b})\right]+\cdots, \\
V \sim\left[v_{0}+2 \lambda E(-\gamma S+\alpha C)\right]+\beta^{-1} \\
\times\left[v_{1}+2 \lambda E\left\{C\left(\gamma g_{1}+\alpha f_{1}\right)+S\left(\alpha g_{1}-\gamma f_{1}\right)\right\}-\frac{E^{2} f_{1}}{\alpha}\right] \\
+\beta^{-2}\left[\begin{array}{l}
v_{2}+2 \lambda E\left\{C\left(\gamma g_{2}+\alpha f_{2}\right)+S\left(\alpha g_{2}-\gamma f_{2}\right)\right\} \\
\left.+\frac{E^{2} \bar{c}}{\alpha}+E^{3}(C \widehat{a}+S \widehat{b})\right]+\cdots,
\end{array}\right.
\end{gathered}
$$

where, for conciseness,

$$
\begin{gathered}
E=e^{-\alpha \eta}, \\
C=\cos \gamma \eta, \\
S=\sin \gamma \eta, \\
\lambda=\left(\alpha^{2}+\gamma^{2}\right)^{-1}, \\
v_{0}=V_{\mathrm{s}}-2 \lambda \alpha,
\end{gathered}
$$

$f_{1}, g_{1}$, and $v_{1}$ are obtained from

$$
\begin{gathered}
\left(3 \alpha^{2}+\gamma^{2}\right) f_{1}+2 \alpha \gamma g_{1}=\lambda\left(\alpha^{2}-\gamma^{2}\right), \\
-2 \alpha \gamma f_{1}+\left(3 \alpha^{2}+\gamma^{2}\right) g_{1}=2 \lambda \alpha \gamma \\
v_{1}=-2 \lambda\left(\gamma g_{1}+\alpha f_{1}\right)+\frac{f_{1}}{\alpha}
\end{gathered}
$$

$\bar{c}$ and $\tilde{c}$ satisfy

$$
\begin{aligned}
& \left(3 \alpha^{2}+\gamma^{2}\right) \bar{c}+2 \alpha \gamma \widetilde{c}=v_{0}\left(\gamma g_{1}+\alpha f_{1}\right)-2 \lambda\left(\alpha^{2}-\gamma^{2}\right) f_{1} \\
& -2 \alpha \gamma \bar{c}+\left(3 \alpha^{2}+\gamma^{2}\right) \tilde{c}=v_{0}\left(\alpha g_{1}-\gamma f_{1}\right)-4 \lambda \alpha \gamma f_{1}
\end{aligned}
$$

$\bar{a}, \bar{b}, \tilde{a}$, and $\tilde{b}$ satisfy

$$
\begin{gathered}
8 \alpha^{2} \bar{a}-6 \alpha \gamma \bar{b}+2 \alpha \gamma \widetilde{a}=-\left(1-4 \lambda \alpha^{2}\right) f_{1}, \\
6 \alpha \gamma \bar{a}+8 \alpha^{2} \bar{b}+2 \alpha \gamma \widetilde{b}=\frac{\left(1-4 \lambda \alpha^{2}\right) \gamma f_{1}}{\alpha}-2 g_{1}, \\
-2 \alpha \gamma \bar{a}+8 \alpha^{2} \widetilde{a}-6 \alpha \gamma \widetilde{b}=-2\left(1-2 \lambda \alpha^{2}\right) g_{1}+\frac{\gamma f_{1}}{\alpha}, \\
-2 \alpha \gamma \bar{b}+6 \alpha \gamma \widetilde{a}+8 \alpha^{2} \widetilde{b}=-4 \lambda \alpha \gamma g_{1}+f_{1},
\end{gathered}
$$

$\widehat{a}$ and $\widehat{b}$ satisfy

$$
\begin{aligned}
& 3 \alpha \widehat{a}-\gamma \widehat{b}=2 \bar{a}, \\
& \gamma \widehat{a}+3 \alpha \widehat{b}=2 \bar{b},
\end{aligned}
$$

and finally $f_{2}, g_{2}$, and $v_{2}$ are obtained from

$$
\begin{aligned}
& f_{2}=-\bar{c}-\bar{a}, \\
& g_{2}=-\widetilde{c}-\tilde{a},
\end{aligned}
$$$$
v_{2}=-2 \lambda\left(\gamma g_{2}+\alpha f_{2}\right)-\frac{\bar{c}}{\alpha}-\widehat{a}
$$ 
Of interest are the radial and azimuthal components of the shear stress at the surface, as well as the far-field speed. They are represented, respectively, by

$$
\begin{aligned}
F^{\prime}(0) & \sim-\alpha+\beta^{-1}\left(\alpha f_{1}+\gamma g_{1}\right) \\
& +\beta^{-2}\left(-\alpha f_{2}+\gamma g_{2}-2 \alpha \bar{c}-3 \alpha \bar{a}+\gamma \bar{b}\right)+\cdots, \\
G^{\prime}(0) & \sim-\gamma+\beta^{-1}\left(\alpha g_{1}-\gamma f_{1}\right) \\
& +\beta^{-2}\left(-\alpha g_{2}-\gamma f_{2}-2 \alpha \widetilde{c}-3 \alpha \widetilde{a}+\gamma \widetilde{b}\right)+\cdots, \\
& V(\infty) \sim v_{0}+\beta^{-1} v_{1}+\beta^{-2} v_{2}+\cdots=V_{\infty} .
\end{aligned}
$$

The expansion for the pressure can be obtained from

$$
Q=-2 F+\frac{1}{2} \beta^{-1}\left(V_{\infty}^{2}-V^{2}\right)
$$

which is the result of integrating (4d) and the use of (4a) and condition (4j). This is not given here, for brevity.

The previously mentioned expansions describe how the flow behaves as $\beta \sim \infty$. They reveal a sinusoidal behavior that dies out exponentially as we move away from the surface. This behavior is solely due to the Hall effect, which is also responsible for the presence of the azimuthal velocity component $G$ (see Appendix).

\section{Results and Discussion}

The asymptotic expansions obtained previously are tested against corresponding numerical results. The problem described by (4a), (4b), (4c), (4d), (4e), (4f), (4g), (4h), (4i), and $(4 \mathrm{j})$ is solved numerically in double precision, using Keller's two-point, second-order accurate, finite-difference scheme [12]. A uniform step size $\Delta \eta=0.01$ is used on a finite domain $0 \leq \eta \leq \eta_{\infty}$. The value of $\eta_{\infty}$ is chosen sufficiently large in order to insure the asymptotic satisfaction of the farfield conditions $(4 \mathrm{~h})-(4 \mathrm{j})$. (As pointed out by Pantokratoras [13], a small value of $\eta_{\infty}$ can lead to erroneous results.) The nonlinear terms are quasi-linearized, and an iterative procedure is implemented, terminating when the maximum errors in $F^{\prime}(0), G^{\prime}(0)$, and $V(\infty)$ become less than $10^{-10}$.

The numerical results exhibit the attenuating sinusoidal behavior predicted by the asymptotic analysis. This is clearly illustrated in Figure 1 showing the radial and azimuthal velocity profiles $F(\eta)$ and $G(\eta)$, when $\beta=10, m=10, n=1.2$, and $V_{s}=20$, with $\eta_{\infty}=350$. However, at such low value of $\beta$, a fixed period of oscillation is not sustained. When $\beta$ is increased to 100 , the $F$ and $G$ profiles, respectively, cross the zero line first at $\eta \approx 7.98$ and 15.67 then at $\eta \approx 23.37$ and 31.10. The two profiles cross the zero line several times later but with much smaller magnitudes, maintaining the same period $\tau \approx 30.9$, all through.

How quantitatively useful the asymptotic expansions of the previous section can be is next investigated. To generate numerical values, for given $\beta, m, n$, and $V_{s}$, we need to determine the coefficients $\alpha, \gamma, \lambda, v_{0}, f_{1}, g_{1}, v_{1}, \ldots$, and so forth. To that end, we use expansions (7a) and (7b) as well

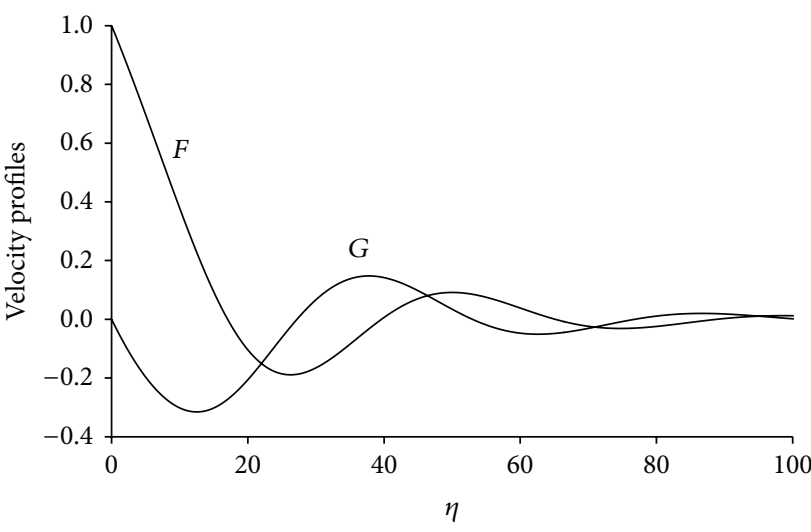

FIGURE 1: Velocity profiles, $\beta=20, m=10, n=1.2$, and $V_{s}=20$.

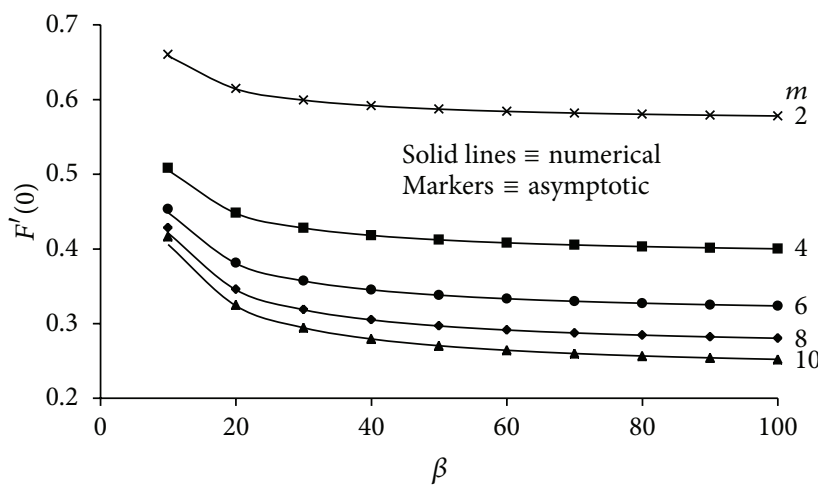

FIGURE 2: Radial surface shear $F^{\prime}(0)$ versus $\beta$, with different values of $n ; m=1, V_{s}=0$.

as (8a), (8b), (8c), (8d), (8e), (8f), (8g), (8h), (8i), (8j), (8k), $(8 \mathrm{l}),(8 \mathrm{~m}),(8 \mathrm{n}),(8 \mathrm{o}),(8 \mathrm{p}),(8 \mathrm{q}),(8 \mathrm{r})$, and $(8 \mathrm{~s})$ to obtain expansions of these coefficients in form (5). In particular, the coefficients of the expansions which are involved to $\mathcal{O}\left(\beta^{-1}\right)$ are found to be $\alpha_{0}=\left[(1 / 2)\left\{\widehat{n}+\left(\widehat{m}^{2}+\widehat{n}^{2}\right)^{1 / 2}\right\}\right]^{1 / 2}, \gamma_{0}=\widehat{m} / 2 \alpha_{0}$, $\lambda_{0}=\left(\alpha_{0}^{2}+\gamma_{0}^{2}\right)^{-1}, v_{00}=V_{\mathrm{s}}-2 \lambda_{0} \alpha_{0}, \alpha_{1}=-(1 / 2) v_{00}$, $\gamma_{1}=0, \lambda_{1}=-2 \lambda_{0}^{2}\left(\alpha_{0} \alpha_{1}+\gamma_{0} \gamma_{1}\right), v_{01}=-2\left(\lambda_{0} \alpha_{1}+\lambda_{1} \alpha_{0}\right)$, $f_{10}=\lambda_{0}\left(\widehat{n} \theta_{1}-\widehat{m} \theta_{2}\right) / \theta_{3}, g_{10}=\lambda_{0}\left(\widehat{n} \theta_{2}+\widehat{m} \theta_{1}\right) / \theta_{3}$, and $v_{10}=-\lambda_{0}\left(\widehat{n} f_{10}+\widehat{m} g_{10}\right) / \alpha_{0}$, where $\theta_{1}=3 \alpha_{0}^{2}+\gamma_{0}^{2}, \theta_{2}=\widehat{m}$, and $\theta_{3}=\theta_{1}^{2}+\theta_{2}^{2}$. The following expansions are also obtained:

$$
\begin{gathered}
F^{\prime}(0)=-\alpha_{0}+\beta^{-1}\left(-\alpha_{1}+\alpha_{0} f_{10}+\gamma_{0} g_{10}\right)+\mathcal{O}\left(\beta^{-2}\right), \\
G^{\prime}(0)=-\gamma_{0}+\beta^{-1}\left(-\gamma_{1}-\gamma_{0} f_{10}+\alpha_{0} g_{10}\right)+\mathcal{O}\left(\beta^{-2}\right), \\
V(\infty)=v_{00}+\beta^{-1}\left(v_{01}+v_{10}\right)+\mathcal{O}\left(\beta^{-2}\right)
\end{gathered}
$$

Results of the two-term asymptotic expansions (i.e., up to $\left.\mathcal{O}\left(\beta^{-1}\right)\right)$ proved to be in close agreement with the numerical results (with $\eta_{\infty}=40$ ), even at values of $\beta$ as low as 10 . This is illustrated in Figure 2 for $F^{\prime}(0)$ with different values of $m$, in Figure 3 for $-G^{\prime}(0)$ with different values of $n$, and in Figure 4 for $V(\infty)$ with different values of $V_{s}$. 


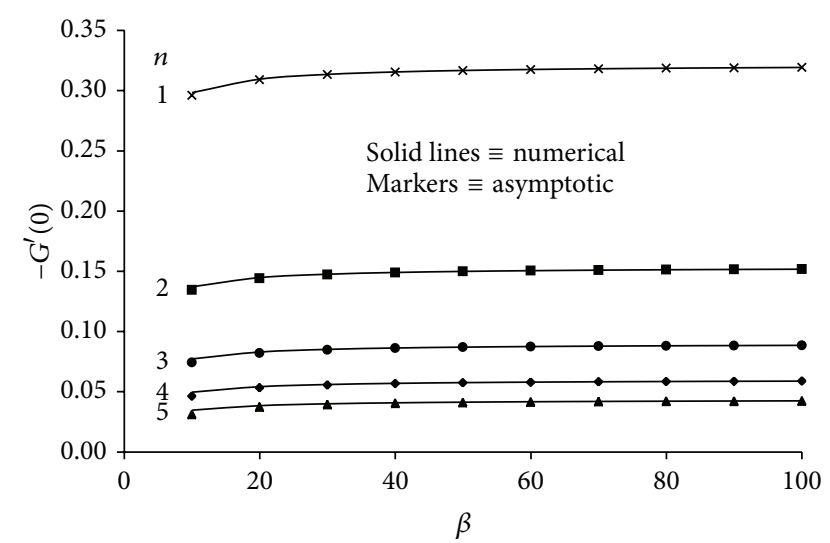

FIGURE 3: Azimuthal surface traction $-G^{\prime}(0)$ versus $\beta$, with different values of $m ; n=1, V_{s}=0$.

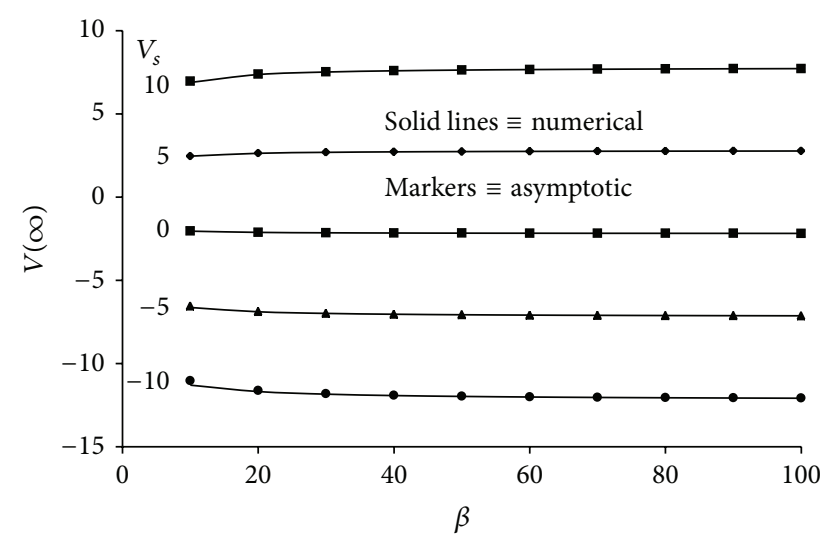

FIGURE 4: Far-field normal velocity $V(\infty)$ versus $\beta$, with different values of $V_{s} ; m=1, n=1$.

The period of the sinusoidal oscillations $\tau=2 \pi / \gamma$ takes the form

$$
\tau=\frac{4 \pi\left[(1 / 2)\left\{\widehat{n}+\left(\widehat{m}^{2}+\widehat{n}^{2}\right)^{1 / 2}\right\}\right]^{1 / 2}}{\widehat{m}}+\mathcal{O}\left(\beta^{-2}\right)
$$

which shows that, to $\mathcal{O}\left(\beta^{-2}\right), \tau$ is independent of $V_{s}$. Partial differentiation of the leading term expression for $\tau$ with respect to $m$ and $n$ reveals that $\tau$ monotonically increases with $n$, while it has a local minimum when $m=n \sqrt{3}$. Typical results are presented in the first six rows of Table 1. They are in excellent agreement with the corresponding averaged numerical results, examples of which, calculated with $\beta=$ $100, V_{s}=0, \eta_{\infty}=100$, are given in the last row of Table 1 , each to be compared with the corresponding (bold) asymptotic value in the same column.

\section{Conclusion}

The limiting behavior of the MHD flow due to a porous stretching disk has been studied, as the magnetic field grows indefinitely, taking into consideration the Hall current and ion-slip effects. Three-term uniformly valid asymptotic
TABLE 1: Period of sinusoidal oscillations $\tau$ for different values of $m$ and $n$.

\begin{tabular}{lcccccc}
\hline$m$ & $n=1.0$ & 1.2 & 1.4 & 1.6 & 1.8 & 2.0 \\
\hline 1 & $\mathbf{1 9 . 5 2 5}$ & 23.0678 & 27.005 & 31.306 & 35.944 & 40.894 \\
2 & 17.871 & $\mathbf{1 9 . 4 7 6}$ & 21.258 & 23.213 & 25.333 & 27.613 \\
3 & 19.109 & 20.146 & $\mathbf{2 1 . 2 8 2}$ & 22.518 & 23.853 & 25.285 \\
4 & 20.731 & 21.510 & 22.354 & $\mathbf{2 3 . 2 6 2}$ & 24.236 & 25.274 \\
5 & 22.379 & 23.013 & 23.692 & 24.418 & $\mathbf{2 5 . 1 8 9}$ & 26.008 \\
$n \sqrt{3}$ & 17.772 & 19.468 & 21.028 & 22.479 & 23.843 & $\mathbf{2 5 . 1 3 3}$ \\
\hline num. $^{\text {a }}$ & $\mathbf{1 9 . 5 4}$ & $\mathbf{1 9 . 4 8}$ & $\mathbf{2 1 . 3 0}$ & $\mathbf{2 3 . 2 8}$ & $\mathbf{2 5 . 2 1}$ & $\mathbf{2 5 . 1 4}$ \\
a $^{\text {Averaged numerical results each to be compared with corresponding (bold) }}$ \\
asymptotic value in the same column.
\end{tabular}

expansions have been derived using parameter straining. The velocity components show sinusoidal behavior that attenuates exponentially, as we move away from the disk. In contrast, when the MHD effect of Hall current is neglected, the exponential decay becomes monotonic. Finite difference solutions have also been calculated. The two-term asymptotic results and the numerical solutions have shown excellent agreement both qualitatively and quantitatively.

\section{Appendix}

The case of the neglect of the Hall and ion-slip effects $\{m=$ $0, n=1$ \} can be obtained by setting $\gamma=0$. The azimuthal velocity component $G$ vanishes identically, while for $F$ and $V$ we get the expansions

$$
\begin{aligned}
F \sim & E+\beta^{-1} \frac{1}{3 \alpha^{2}}\left[E-E^{2}\right]+\beta^{-2} \frac{1}{72 \alpha^{4}} \\
& \times\left[E\left(-8 V_{s} \alpha+23\right)+E^{2}\left(8 V_{s} \alpha-32\right)+E^{3} 9\right]+\cdots,
\end{aligned}
$$

$$
\begin{aligned}
V \sim \frac{1}{\alpha}\left[\left(V_{s} \alpha-2\right)\right. & +2 E]-\beta^{-1} \frac{1}{3 \alpha^{3}}(1-E)^{2} \\
+\beta^{-2} \frac{1}{72 \alpha^{5}}[ & \left(8 V_{s} \alpha-20\right)+E\left(-16 V_{s} \alpha+46\right) \\
+ & \left.E^{2}\left(8 V_{s} \alpha-32\right)+6 E^{3}\right]+\cdots
\end{aligned}
$$

with

$$
\begin{gathered}
F^{\prime}(0) \sim-\alpha+\frac{1}{3 \alpha} \beta^{-1}+\beta^{-2} \frac{1}{36 \alpha^{3}}\left(-4 V_{s} \alpha+7\right)+\cdots, \\
V(\infty) \sim \frac{1}{\alpha}\left(V_{s} \alpha-2\right)-\frac{1}{3 \alpha^{3}} \beta^{-1}+\beta^{-2} \frac{1}{18 \alpha^{5}}\left(2 V_{s} \alpha-5\right)+\cdots,
\end{gathered}
$$

where $\alpha$ is determined from

$$
1 \sim \alpha^{2}+\beta^{-1}\left(V_{s} \alpha-2\right)-\frac{1}{3 \alpha^{2}} \beta^{-2}+\cdots .
$$


This asymptotic relation can be used to determine an expansion for $\alpha$ of form (5), through substituting and equating coefficients of like powers of $\beta$. The result is

$$
\alpha \sim 1+\beta^{-1}\left(1-\frac{1}{2} V_{s}\right)+\beta^{-2}\left(\frac{1}{8} V_{s}^{2}-\frac{1}{3}\right)+\cdots .
$$

It is to be noted that the sinusoidal behavior disappears. Expansions (A.1a) and (A.1b) involve negative exponentials $\left(e^{-i \alpha \eta}, i=1,2, \ldots\right)$ only.

Further, introduction of expansion (A.3b) into expansions (A.1a), (A.1b), (A.2a), and (A.2b), then expanding, leads to expansions that correspond to the expansions of Ariel [5], who treated the case of no mass transfer, $V_{s}=0$, through coordinate straining.

\section{References}

[1] C. Y. Wang, "The three-dimensional flow due to a stretching flat surface," The Physics of Fluids, vol. 27, no. 8, pp. 1915-1917, 1984.

[2] P. D. Ariel, "Generalized three-dimensional flow due to a stretching sheet," Zeitschrift fur Angewandte Mathematik und Mechanik, vol. 83, no. 12, pp. 844-852, 2003.

[3] P. D. Ariel, "Axisymmetric flow due to a stretching sheet with partial slip," Computers and Mathematics with Applications, vol. 54, no. 7-8, pp. 1169-1183, 2007.

[4] P. D. Ariel, "Extended homotopy perturbation method and computation of flow past a stretching sheet," Computers and Mathematics with Applications, vol. 58, no. 11-12, pp. 2402-2409, 2009.

[5] P. D. Ariel, "Computation of MHD flow due to moving boundaries," International Journal of Computer Mathematics, vol. 86, no. 12, pp. 2165-2180, 2009.

[6] P. D. Ariel, T. Hayat, and S. Asghar, "Homotopy perturbation method and axisymmetric flow over a stretching sheet," International Journal of Nonlinear Sciences and Numerical Simulation, vol. 7, no. 4, pp. 399-406, 2006.

[7] T. Hayat, L. Ahmad, and T. Javed, "On comparison of the solutions for an axisymmetric flow," Numerical Methods for Partial Differential Equations, vol. 25, no. 5, pp. 1204-1211, 2009.

[8] T. Hayat and M. Sajid, "Analytic solution for axisymmetric flow and heat transfer of a second grade fluid past a stretching sheet," International Journal of Heat and Mass Transfer, vol. 50, no. 1-2, pp. 75-84, 2007.

[9] A. H. Nayfeh, Perturbation Methods, John Wiley \& Sons, New York, NY, USA, 1973.

[10] G. W. Sutton and A. Sherman, Engineering Magnetohydrodynamics, McGraw-Hill, New York, NY, USA, 1965.

[11] F. B. Hildebrand, Advanced Calculus for Applications, Prentice Hall, Upper Saddle River, NJ, USA, 1976.

[12] H. B. Keller, "Accurate difference methods for linear ordinary differential systems subject to linear constraints," SIAM Journal on Numerical Analysis, vol. 6, no. 1, pp. 8-30, 1969.

[13] A. Pantokratoras, "A common error made in investigation of boundary layer flows," Applied Mathematical Modelling, vol. 33, no. 1, pp. 413-422, 2009. 


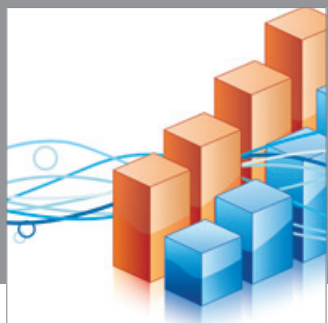

Advances in

Operations Research

mansans

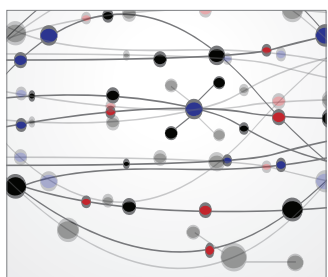

The Scientific World Journal
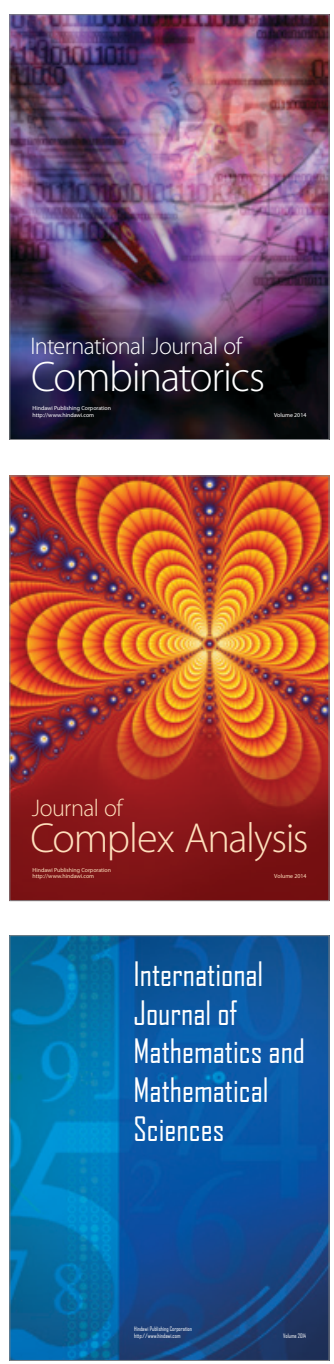
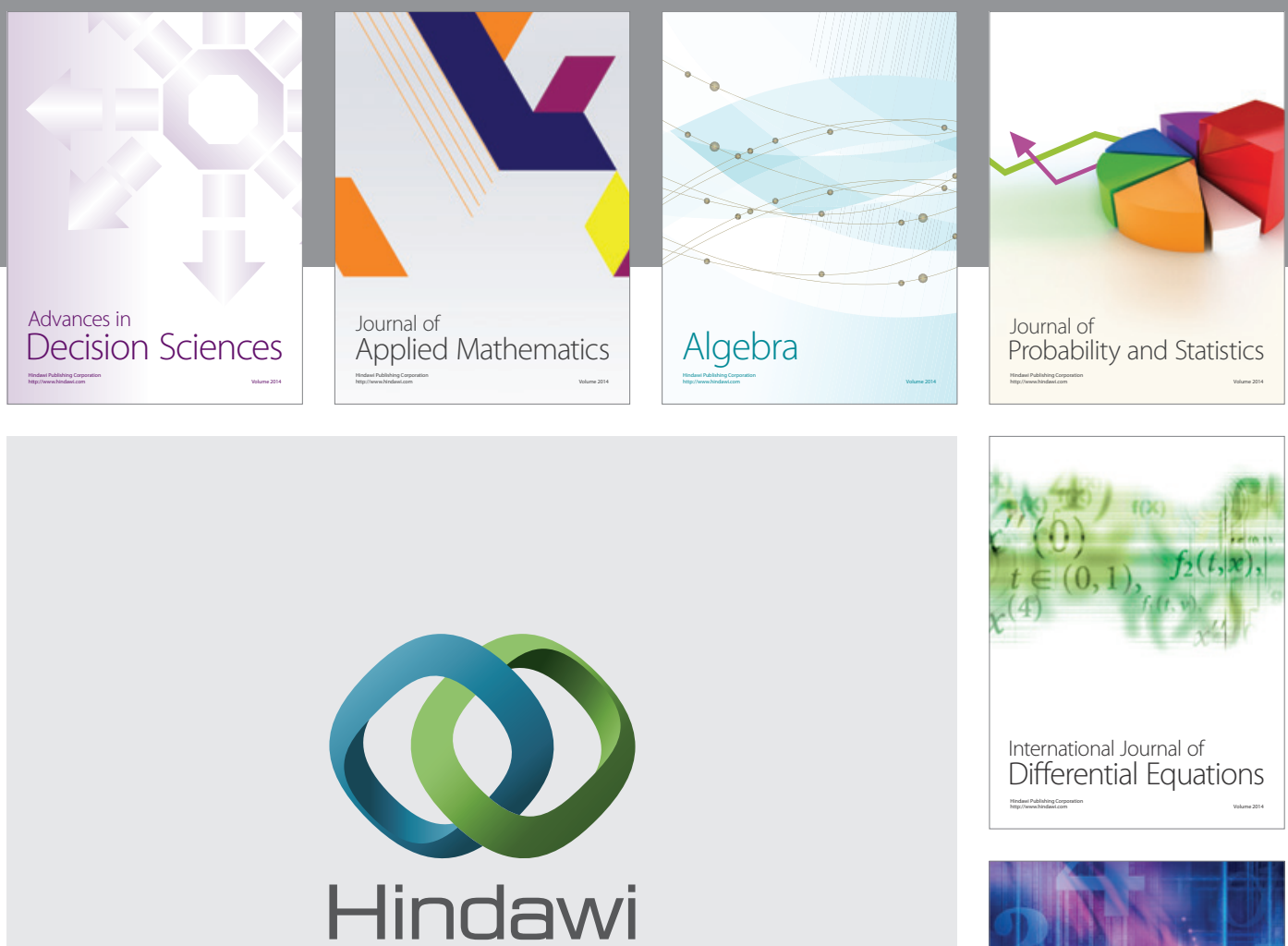

Submit your manuscripts at http://www.hindawi.com
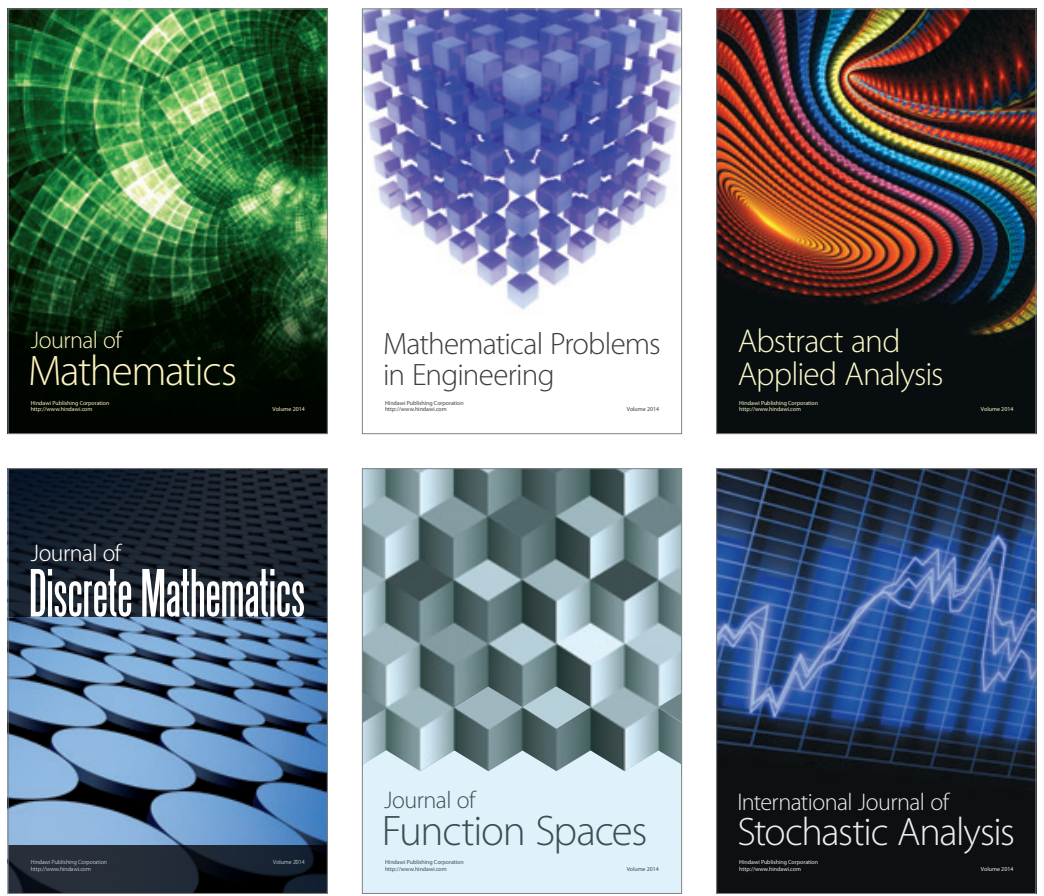

Journal of

Function Spaces

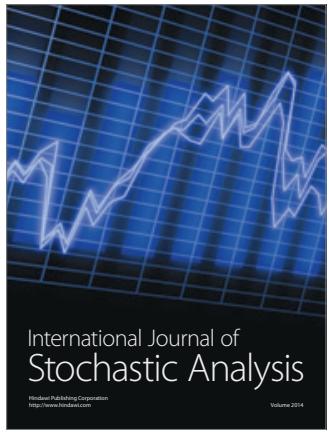

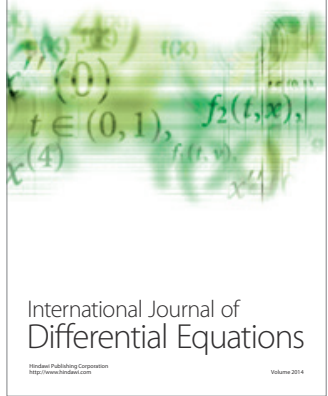
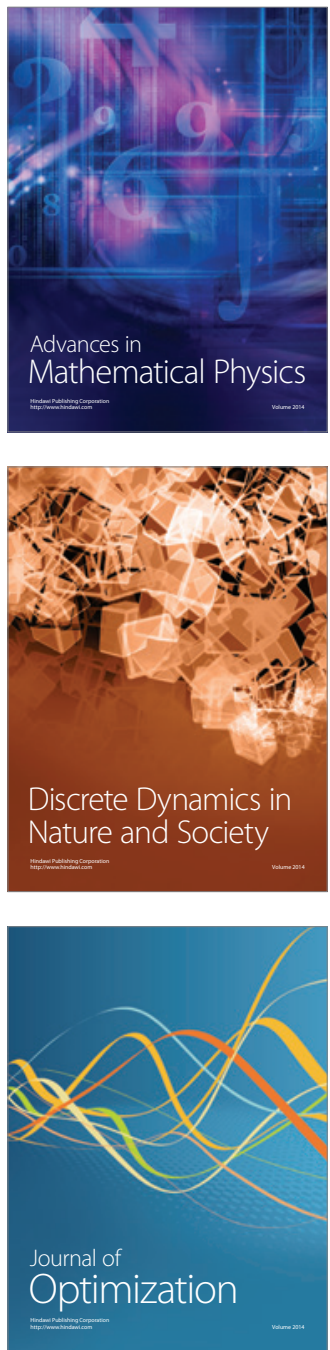